\title{
Intervalos de referência no laboratório clínico
}

\section{Reference ranges in clinical laboratory}

Carlos Eduardo dos Santos Ferreira'; Adagmar Andriolo ${ }^{2}$

unitermos
Intervalo de referência
Valores normais
Laboratório clínico
Normalidade
Intervalos de significância

significância

\section{resumo}

A definição dos intervalos de referência é tarefa desafiadora para todos os laboratórios clínicos. Entre as possibilidades para estabelecimento desses intervalos destacam-se a definição dos próprios intervalos, a validação dos dados constantes das bulas reagentes e a utilização das informações disponíveis na literatura. A criação de intervalos próprios é a escolha mais desejável para a grande maioria dos testes, pois reflete a condição da população para a qual os eles serão aplicados no dia-a-dia, sendo, porém, a mais trabalhosa e onerosa. A validação dos intervalos fornecidos pelas bulas reagentes e a aplicação dos dados fornecidos pela literatura parecem ser as opções mais freqüentemente utilizadas em nosso meio. A escolha de intervalos adequados de referência é fundamental para que os laboratórios clínicos forneçam informações fidedignas e que os clínicos possam interpretar corretamente os resultados e optar pelas melhores condutas diante da população assistida.

\section{abstract}

The definition of reference ranges is a challenging task to all clinical laboratories. It is particularly worth mentioning the definition of their own ranges, the validation of data on reagent directions and the use of information available in medical literature as possibilities for the establishment of these ranges. The creation of their own ranges is undoubtedly the most desirable choice in most tests, inasmuch as it reflects the condition of the population on whom they will be applied on a daily basis, yet it is the most laborious and onerous. The validation of ranges offered by the reagent directions and the use of data from medical literature seem to be the most commonly used options in our country. The choice of appropriate reference ranges is essential so that clinical laboratories offer reliable information and physicians interpret the results correctly and choose the best approach towards the assisted population. key words

Reference ranges

Normal values

Clinical laboratory

Normality

Significance range

1. Médico patologista clínico; mestre pela Escola Paulista de Medicina da Universidade Federal de São Paulo (UNIFESP); médico do Instituto Dante Pazzanese de Cardiologia/Fundação Adib Jatene; assessor médico do Laboratório Clínico no Hospital Israelita Albert Einstein.

2. Médico patologista clínico; doutor livre-docente do Departamento de Medicina da Escola Paulista de Medicina da UNIFESP; assessor médico de Fleury Medicina e Saúde. 


\section{Introdução}

Com o avanço tecnológico ocorrido nas últimas décadas, os testes laboratoriais também sofreram, e continuam sofrendo, mudanças significativas. O impacto dessas mudanças precisa ser analisado para que o clínico possa utilizar com critérios as informações oriundas dos laudos de exames laboratoriais ${ }^{(12)}$.

O primeiro fato a ser destacado é o número de analitos atualmente disponíveis em um laboratório clínico de grande porte. Estima-se que, entre pesquisa e rotina, estejam disponíveis aproximadamente 7.500 diferentes tipos de exames distribuídos nas diferentes áreas de atuação do laboratório. Uma segunda mudança diz respeito à acessibilidade aos recursos laboratoriais por populações específicas ${ }^{(20)}$. A cada dia um número maior de indivíduos saudáveis tem sido submetido a baterias de testes com finalidades preventivas ou, mesmo, apenas administrativas. São os chamados exames de triagem (check-up) e os exames periódicos.

Para que o resultado de um teste laboratorial se transforme em informação fidedigna e seja útil a diagnóstico, monitoramento, estadiamento ou predição de alguma doença, ele precisa ser analisado criteriosamente em vários aspectos. A consistência metodológica, demonstrada já na fase inicial do seu desenvolvimento na pesquisa básica; suas praticidade e viabilidade quando da implantação na rotina clínica e sua robustez diagnóstica no momento em que se torna disponível para a prática clínica são algumas das características indispensáveis ${ }^{(7)}$. Ao ser incorporado ao rol de exames disponibilizados, além dessas características, o teste deve ter sido suficientemente avaliado em diferentes populações e o intervalo de referência ou, mais genericamente, os intervalos de significância devem ter sido estabelecidos, com a segurança possível. A partir desses intervalos a interpretação dos resultados se tornará possível e útil.

Da correta e abrangente definição dos intervalos depende a importância que os testes laboratoriais assumem na conduta clínica. Um estudo já clássico demonstrou que pelo menos $70 \%$ das decisões clínicas no ambiente hospitalar implicando, por exemplo, a conduta de admissão ou não, alta e modificação no esquema terapêutico são altamente dependentes dos resultados dos testes laboratoriais e, conseqüentemente, da interpretação dos intervalos de significância ${ }^{(9)}$.

\section{Discussão}

A Organização Mundial de Saúde (OMS), a Federação Internacional de Química Clínica (IFCC) e o Instituto de
Padronização Clínica e Laboratorial (CLSI) definem valor de referência como um valor (resultado) obtido pela observação ou mensuração quantitativa de um analito em um indivíduo selecionado, com base em critérios bem definidos. O esquema a seguir mostra, representativamente, de onde provêm os intervalos de referência (Figura) ${ }^{(4)}$.

No laboratório clínico, o primeiro passo para a determinação dos intervalos de referência é definir de quem é a responsabilidade dessa definição. A Joint Comission on Accreditation of Healthcare Organizations (JCAHO) $)^{(15)}$ e o College of American Pathologists (CAP) ${ }^{(6)}$ definem que a responsabilidade do estabelecimento dos intervalos referenciais é do diretor do laboratório. A lei que rege os laboratórios americanos, definida em 1988 (CLIA 88) ${ }^{(5)}$ orienta como os laboratórios devem criar e avaliar, periodicamente, seus valores de referência. No Brasil, a legislação (RDC 302) da Agência Nacional de Vigilância Sanitária (ANVISA) $)^{(3)}$ e o Programa de Acreditação de Laboratórios Clínicos (PALC), da Sociedade Brasileira de Patologia Clínica/Medicina Laboratorial (SBPC/ML) definem apenas que o laboratório deve possuir esses valores e fornecê-los no laudo dos exames.

Agência Nacional de Vigilância Sanitária (RDC 302): - 6.3.3 (m) - o laudo deve conter valor de referência, limitações técnicas e dados para interpretação.

Programa de Acreditação e Laboratórios Clínicos (PALC):

- $4.5(\mathrm{~m})$ o laboratório deve possuir valores de referência e valores críticos;

- 10.1 (m) o laudo deve conter valor de referência e/ou dados para interpretação.

Para iniciar um estudo de como definir o intervalo de referência, o laboratório deve realizar ampla revisão da

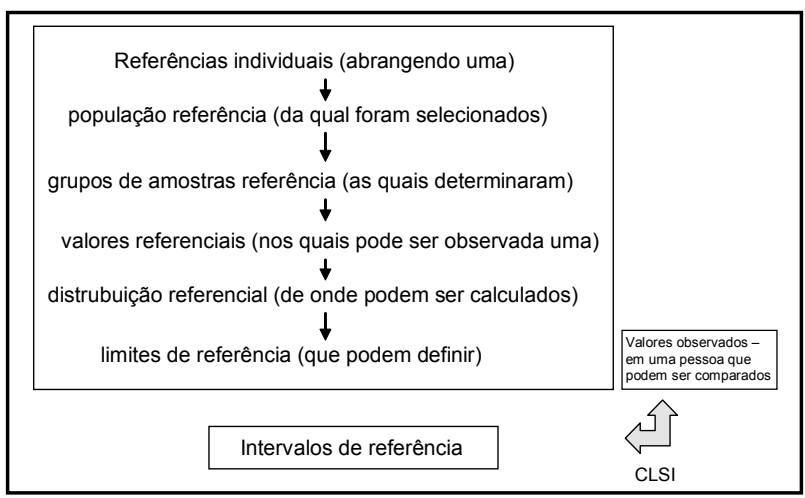

Figura - Esquema básico da determinação de intervalos de referência, segundo o CLSI 
literatura nacional e internacional perante cada parâmetro a ser avaliado. O passo seguinte é definir se o laboratório irá criar seus próprios valores, validar dados de bulas reagentes ou utilizar aqueles de dados disponíveis na literatura. Vale a pena salientar que cada teste deve ser analisado separadamente e que um mesmo laboratório pode dispor dos três tipos de ferramentas para definir seus intervalos de referência.

A determinação de intervalos de referência próprios é mais trabalhosa e onerosa do que as outras opções. Para essa determinação devem ser avaliadas as variáveis biológicas, definidas como todos os fatores que podem interferir individualmente em uma determinada condição, promovendo variação intra-indivíduo ${ }^{(25)}$. Essas variáveis podem ser classificadas como ritmos biológicos, fatores constitucionais e fatores extrínsecos. Os ritmos biológicos mais significativos em relação aos exames laboratoriais são três: circadiano, ultradiano e infradiano. O ritmo circadiano é definido como as variações na concentração de determinada substância em um período de 24 horas. Por exemplo, a concentração de cortisol sérico apresenta pico nas primeiras horas da manhã e cai ao longo do dia ${ }^{(10,17)}$. O ritmo ultradiano inclui variações ocorridas em curtos períodos de tempo, em geral, minutos. São exemplos as substâncias liberadas para a circulação por pulsos. O ritmo infradiano caracteriza as variações que ocorrem em um período de 30 dias. Os hormônios sexuais femininos, por exemplo, como os hormônios luteinizante e folículo-estimulante, apresentam esse comportamento(2).

Os fatores constitucionais incluem o gênero, a idade e a variedade genética. Os principais fatores extrínsecos são a postura; a realização de exercícios físicos; a dieta, compreendendo, além do tempo de jejum, o teor da alimentação habitual, o uso de cafeína e bebidas alcoólicas; utilização de drogas com fins terapêuticos ou não e gravidez ${ }^{(17)}$.

É válido ressaltar que a importância dos fatores constitucionais pode variar ao longo do tempo ao se considerar uma doença ou um conjunto de doenças em particular. Isso pode ser bem evidenciado, por exemplo, nas principais doenças do sistema circulatório (infarto agudo do miocárdio e acidente vascular cerebral). Essas doenças são, atualmente, as principais causas de mortalidade no Brasil e em grande parte do mundo. Há aproximadamente 30 anos, o gênero masculino e a idade acima dos 40 anos eram praticamente obrigatórios para a ocorrência desses eventos. Com o passar dos anos, a idade vem se reduzindo, e a prevalência de infarto em jovens é cada vez maior. Igualmente, a prevalência da doença no sexo feminino sofreu elevação significativa, em parte devido às alterações dos hábitos alimentares, ao aumento do tabagismo na população feminina e de jovens e ao ingresso da mulher no mercado de trabalho, elevando o nível de estresse e sedentarismo ${ }^{(31)}$.

A interferência dos fatores extrínsecos constitui não só um problema para a definição dos intervalos de referência a ser equacionado, mas um desafio para os laboratórios, exigindo redobrada atenção em relação às condições do paciente e aos protocolos de padronização dos procedimentos de atendimento do paciente e coleta de amostras ${ }^{13,}$ 16). A atividade física pode ser utilizada para exemplificar a dificuldade de se avaliar e minimizar a interferência dos fatores extrínsecos. O exercício físico pode ser realizado em suas diversas formas e intensidades, de uma simples caminhada à realização de uma maratona. A variação decorrente nos resultados dos exames laboratoriais pode ser evidenciada, por exemplo, com o aumento da leucometria e a liberação de catecolaminas após uma caminhada leve ou na concentração sérica de creatina quinase, mioglobina, troponina I, creatinina, entre outros, após uma maratona. Outro fator complicador na avaliação da intensidade da interferência da atividade física sobre os diversos parâmetros é a variabilidade causada pelo condicionamento físico do indivíduo ${ }^{(28)}$. A interpretação dos resultados dos principais eletrólitos é outro importante exemplo. Dispondo de informações pré-analíticas, extrínsecas e fatores constitucionais, como a idade do paciente, a avaliação dos resultados desses analitos se torna mais criteriosa ${ }^{(14)}$.

Feita a avaliação global das variáveis biológicas, o próximo passo para a determinação dos intervalos de referência é a escolha da amostragem a ser avaliada. O número de indivíduos a serem avaliados é bastante controverso na literatura variando de 30 a 700 amostras. O CLSI (C24A3) e a IFCC preconizam a utilização de, no mínimo, 119 sujeitos para a utilização de testes não-paramétricos. Para a utilização de testes paramétricos, a distribuição deve ser normal e a amostra deve conter mais de 30 indivíduos. Os critérios pré-analíticos como tempo de jejum, dieta habitual, postura, tempo de garroteamento, grau de atividade física, tipo de tubos, tipo de amostra, processamento, transporte e conservação da amostra devem estar bem estabelecidos e padronizados. Os procedimentos analíticos, incluindo método, equipamentos e conjuntos diagnósticos, também devem ser cuidadosamente calibrados e harmonizados, se for o caso. Características da metodologia, como precisão, exatidão, sensibilidade analítica, linearidade, reprodutibilidade e potenciais interferentes devem ser conhecidas e, na medida do possível, estar sob controle estrito ${ }^{(16,30)}$. 
Após a obtenção dos dados, a análise estatística pode ser feita com testes paramétricos ou não-paramétricos, na dependência da natureza e da distribuição deles. No teste não-paramétrico preconizado pelo CLSI, deve ser utilizado o percentil 97,5 para a maioria dos parâmetros, porém existem numerosas exceções, como o caso das troponinas (T e l), para os quais deve ser utilizado o percentil $99^{(29)}$. Atenção especial deve ser dada aos outliers, indivíduos que apresentam resultados muito discrepantes em relação aos demais componentes do grupo. Como regra, obedecidas as grandezas numéricas totais já referidas, até $10 \%$ de uma amostragem podem ser desprezados por conta de outliers. Um dos recursos para a caracterização de um dado como outlier é o teste de Dixon, utilizando a relação de $D / R$, na qual:

- D é a diferença entre o maior valor e o segundo maior valor ou o menor valor do segundo menor valor;

- R é a diferença entre o maior valor e o menor valor, também identificado como amplitude;

- se $D / R>0,33$ o dado pode ser considerado outlier.

A outra opção válida para a definição dos intervalos de referência é a validação de valores especificados na bula do conjunto diagnóstico. Para realizar essa validação o CLSI preconiza testar 20 indivíduos considerados saudáveis. Das amostras, apenas duas (10\%) podem fornecer resultados fora do intervalo referido pela bula reagente. Caso contrário, as dosagens devem ser repetidas e, se o erro se repetir, os valores de bula não poderão ser utilizados ${ }^{(23,24)}$. O CAP preconiza que os valores de referência deverão ser criados ou validados conforme estabelecido pela literatura. Os valores deverão ser revistos a cada mudança de metodologia, alteração na população avaliada e/ou implantação de um novo analito no menu de exames do laboratório.

Para efetuar as avaliações estatísticas, os cálculos matemáticos manuais podem ser utilizados, porém, em geral, são trabalhosos. Programas de computador amplamente disponíveis no mercado são opções seguras e práticas. Entre eles, podem ser referidos o Statistical Package for the Social Sciences (SPSS ${ }^{\circledast}$ ), o Office ${ }^{\circledR}$ (Excel) e o EP Evaluator ${ }^{\circledR}$, entre outros.

Diante da dificuldade de serem determinados e validados intervalos significativos para populações específicas, como a pediátrica e a geriátrica, podem ser utilizados dados fornecidos pela literatura, com base em estudos que definam valores referenciais para essas populações específicas. Para a validação é importante que as características da população e a metodologia utilizada pelo estudo sejam compatíveis $^{(26)}$.
Para a população pediátrica já podem ser encontrados numerosos trabalhos que orientem a escolha de intervalos referenciais ${ }^{(18,27)}$, mas para a população geriátrica os estudos ainda são escassos e não há consenso sobre quais testes devem ser reportados com intervalos referenciais específicos ${ }^{(8)}$.

Certos analitos, como a glicose, a proteína C-reativa, os lipídeos e as lipoproteínas, possuem valores referenciais provenientes de estudos clínicos que utilizam grande amostragem e realizam seguimento clínico dos indivíduos por variados períodos de tempo. Em geral, esses intervalos são consistentes, utilizados pela maioria dos laboratórios e com ampla disseminação nas publicações científicas e na mídia aberta, mas os critérios de definição dos limites são, em geral, clínicos, com base na ocorrência ou não de um evento em especial ou na predisposição ou não para determinada doença e não na distribuição dos valores obtidos ${ }^{(20,}$ 1). Exemplificando, $200 \mathrm{mg} / \mathrm{dl}$, considerado limite superior adequado para colesterol total corresponde ao percentil 50 . Esse procedimento permite a criação de um conceito de risco de ter ou desenvolver determinada doença, que seria maior ou menor na dependência de quanto o resultado de um indivíduo se distancia do limite considerado adequado. Dessa forma, alguém com colesterol total de $300 \mathrm{mg} / \mathrm{dl}$ teria, potencialmente, maior risco de desenvolver doença aterosclerótica em relação a outro indivíduo que apresentasse todas as demais condições iguais, mas com colesterol total de $240 \mathrm{mg} / \mathrm{dl}$, por exemplo(21).

A maioria dos testes laboratoriais apresenta intervalos de referência para sua avaliação, porém para alguns parâmetros existem outras formas para diferenciação entre o "normal" e o "patológico". Entre essas formas, destacam-se a mediana, a distribuição das médias, múltiplos da média ou da mediana e percentis da distribuição referência. Para escolher a forma mais adequada de apresentação do resultado, é importante realizar uma revisão de literatura. A dosagem de alfa-fetoproteínas no soro materno e a definição de intervalos significativos em múltiplos da mediana, para cada idade gestacional, na avaliação do risco de defeito do tubo neural do feto é um bom exemplo de uma forma bem particular de interpretar os resultados ${ }^{(26)}$.

Outros testes, principalmente os imunológicos, microbiológicos e de biologia molecular, podem necessitar que o resultado seja expresso de forma mais específica, ou seja, como positivo ou negativo, presente ou ausente. Entretanto, em algumas situações, o resultado de um teste dessa natureza pode não ser conclusivo o suficiente. A presença de janelas imunológicas, interferências medicamentosas, 
associação de doença e os diferentes graus de integridade do sistema imune são condições nas quais o resultado final pode não representar o real estado de saúde do indivíduo, gerando dúvidas na sua interpretação.

O cenário atual nos Estados Unidos para os valores de referência foi descrito em um artigo avaliando sete parâmetros (potássio, hemoglobina, plaquetas, TSH, cálcio magnésio e tempo tromboplastina parcial ativado [TTPA]) fornecidos por 163 laboratórios clínicos. Para a população adulta, aproximadamente $50 \%$ dos laboratórios utilizam seus próprios intervalos de referência. Quando avaliada a população pediátrica, esse percentual se reduz para $25 \%$. As demais formas de aplicação de intervalos de referência nos laboratórios pesquisados incluíram validação de valores de bula, utilização de dados disponíveis na literatura, utilização de valores de outros laboratórios (com e sem validação) e recomendação da equipe do laboratório. Para exemplificar a complexidade da determinação dos intervalos, o mesmo estudo demonstrou que o intervalo de referência para a hemoglobina, em homens adultos, variou de 11,5 a $14,8 \mathrm{~g} / \mathrm{dl}$ em um laboratório para 15 a 18,1 g/dl em outro(11).

No Brasil, pouco se sabe como estão estruturados os laboratórios clínicos em relação à determinação dos intervalos de referência. Dados iniciais sugerem que uma parcela significativa deles utiliza os intervalos sugeridos nas bulas dos conjuntos diagnósticos e os encontrados na literatura internacional. Infelizmente, nem sempre essas duas fontes são adequadas para a realidade nacional, dadas as características da população brasileira, sendo desejável um esforço para a aplicação de intervalos próprios.

\section{Conclusão}

A definição dos intervalos de referência é tarefa desafiadora para todos os laboratórios clínicos. O direcionamento de como o laboratório procederá (se criará seus próprios intervalos, validará os dados constantes das bulas reagentes ou utilizará as informações da literatura) é o passo inicial para essa definição. A criação de intervalos próprios é, sem dúvida, a melhor escolha para a grande maioria dos testes, uma vez que reflete a condição da população para a qual os testes serão aplicados no dia-a-dia, sendo, porém, a mais trabalhosa e onerosa. A validação dos intervalos fornecidos pelas bulas reagentes é a opção menos custosa, em conjunto com a avaliação criteriosa da literatura.

Essa definição se torna fundamental para que os laboratórios clínicos forneçam informações fidedignas e que os clínicos possam interpretar corretamente os resultados e optar por condutas adequadas à população assistida.

\section{Referências}

1. AMERICAN DIABETES ASSOCIATION (ADA). Standards of medical care in diabetes. Diabetes Care, v. 28, suppl. 1, 2005.

2. ANDRIOLO, A.; ROCHA, M. H. Características e interpretação dos resultados dos exames laboratoriais. In: ANDRIOLO, A.; CARRAZA, F. R. Diagnóstico laboratorial em pediatria. 2 ed. São Paulo: Sarvier, 2007. Cap. 1; p. 3-13.

3. BRASIL. Ministério da Saúde. Agência Nacional de Vigilância Sanitária (ANVISA). Dispõe sobre regulamentação técnica para funcionamento de laboratórios clínicos. Resolução da Diretoria Colegiada - RDC n³ 302, 2005.

4. CLINICAL AND LABORATORY STANDARDS INSTITUTE. How to define and determine reference intervals in the clinical laboratory. Document C 28-A2, 2000.

5. CLINICAL LABORATORY IMPROVEMENT AMENDMENTS of 1988 (CLIA), 42 CFR $\$ 493.1253$ (b) (1) (ii) (2003)

6. COLLEGE OF AMERICAN PATHOLOGISTS. Commission on Laboratory Accreditation Inspection Checklist. Laboratory general. Northfield, IL: CAP, 1998.

7. DYBKAER, R.; SOLBERG, H. E. Approved recommendations (1987) on the theory of reference values. Part6. Presentation of observed values related to reference values. J Clin Chem Clin Biochem, v. 25, p. 657-62, 1987.

8. FAULKNER, W.; MEITES, S. Geriatric clinical chemistry reference values. Washington, DC: AACC Press, 1994.

9. FORSMAN, R. W. Why is the laboratory an afterthought for managed care organizations? Clin Chem, v. 42, n. 5 , p. 813-6, 1996.

10. FRASER, C. G. Biological variation in clinical chemistry, an update: collated data, 1988-1991. Arch Pathol Lab Med, v. 116, p. 916, 1991

11. FRIEDBERG, R. C. et al. The origin of reference intervals. A College of American Pathologists Q-Probes study of "normal ranges" used in 163 clinical laboratories. Arch Pathol Lab Med, v. 131, n. 3, p. 348-57, 2007.

12. GALLAHER, M. P. et al. The impact of calibration error in medical decision making: final report. Gaithersburg, MD: National Institute of Standards and Technology; 2004.

13. HOWANITZ, P. J. Quality assurance measurements in department of pathology and laboratory medicine. Arch Pathol Lab Med, v. 114, p. 1131-5, 1990. 
14. HOWANITZ, P. J.; CEMBROWSKI, G. S. Post analytical quality improvement: a College of American Pathologists Q-Probes study of elevated calcium results in 525 institutions. Arch Pathol Lab Med, v. 124, p. 504-10, 2000.

15. JOINT COMMISSION ON ACCREDITATION OF HEALTHCARE ORGANIZATIONS. 1998-99 Comprehensive accreditation manual for pathology and clinical laboratory services. Oakbrook Terrace, IL: JCAHO, 1998.

16. KLEIN, G.; JUNGE, W. Creation of the necessary analytical quality for generating and using reference intervals. Clin Chem Lab Med, v. 42, p. 851-7, 2004.

17. McCLATCHEY, K. D. Clinical laboratory medicine. In: HOLMES, A. C. The interpretation of laboratory tests. $2^{\text {nd }}$ ed. Philadelphia: Lippincott Williams \& Wilkins, 2002. Sec. 4; p. 97-122.

18. MEITES, S. Pediatric clinical chemistry reference (normal) values. $3^{\text {rd }}$ ed. Washington, DC: AACC Press, 1989.

19. PETITCLERC, C.; SOLBERG, H. E. Approved recommendation (1987) on the theory of reference values. Part 2. Selection of individuals for the production of reference values. J Clin Chem Clin Biochem, v. 25, p. 639-44, 1987.

20. RIDKER, P. M. Clinical application of C-reactive protein for cardiovascular disease detection and prevention. Circulation, v. 107, p. 363-9, 2003.

21. SOCIEDADE BRASILEIRA DE CARDIOLOGIA. I/I Diretrizes Brasileiras sobre dislipidemias e diretrizes de prevenção da aterosclerose do Departamento de Aterosclerose da Sociedade Brasileira de Cardiologia. Arq Bras Cardiol, v. 77, sup. III, p. 10-1, 2001.

22. SOLBERG, H. E. Approved recommendation (1986) on the theory of reference values. Part 1 . The concept of reference values. Clin Chem Acta, v. 167, p. 1118, 1987.

23. SOLBERG, H. E. Approved recommendations (1987) on the theory of reference values. Part 5. Statistical treatment of collected reference values. Determination of reference limits. J Clin Chem Clin Biochem, v. 25, p. 645-56, 1987.

24. SOLBERG, H. E.; STAMM, D. Approved recommendation on the theory of reference values. Part 4. Control of analytical variation in the production, transfer, and application of reference values. Eur J Clin Chem Clin Biochem, v. 29, p. 531-5, 1991.

25. SOLBERG, H. E; PETITCLERC, C. Approved recommendation (1988) on the theory of reference values. Part 3. Preparation of individuals and collection of specimens for the production of reference values. Clin Chem Acta, v. 177, S1-S2, 1988.

26. SOLBERG, H. R. Establishment and use of reference values. In: BURTIS, C. A.; ASHWOOD, E. R.; BRUNS, D. E. (editors). Tietz textbook of clinical chemistry and molecular diagnostics. $4^{\text {th }}$ ed. Philadelphia: Elsevier Saunders; 2006. p. 425-48.

27. SOLDIN, S. J.; BRUGNARA, C.; WONG, E. C. Pediatric reference ranges. $4^{\text {th }}$ ed. Washington, DC: AACC Press, 2003.

28. STEELE, B. W. et al. Total long-term within-laboratory precision of cortisol, ferritin, thyroxine, free thyroxine, and thyroid-stimulating hormone assays based on a College of American Pathologists fresh frozen serum study: do available methods meet medical needs for precision? Arch Pathol Lab Med, v. 129, p. 318-22, 2005.

29. THYGESEN, K. et al. Universal definition of myocardial infarction. European Heart Journal, v. 28, p. 252538, 2007.

30. VALENSTEIN, P. Quality management in clinical laboratories: promoting patient safety through risk reduction and continuous improvement. Chicago, III: College of American Pathologists, 2005.

31. YUSUF, S. et al. Effect of potentially modifiable risk factors associated with myocardial infarction in 52 countries (the INTERHEART study): case-control study. The Lancet, v. 364, n. 9438, 2004. 